\title{
Induction of the Vitamin D Receptor Attenuates Autophagy Dysfunction- Mediated Cell Death Following Traumatic Brain Injury
}

\author{
Changmeng Cuia,b Jianzhong Cui ${ }^{c}$ Feng Jin ${ }^{a}$ Ying Cuic Ran Lid,e \\ Xiaohua Jiang ${ }^{\mathrm{d}, \mathrm{e}}$ Yanxia Tiand,e Kaijie Wang ${ }^{\mathrm{c}}$ Pei Jiang ${ }^{\mathrm{b}}$ Junling Gaod,e
}

\begin{abstract}
aDepartment of Neurosurgery, Affiliated Hospital of Jining Medical University, Jining, 'bInstitute of Clinical Pharmacy and Pharmacology, Jining First People's Hospital, Jining Medical University, Jining, 'Tangshan Gongren Hospital, Tangshan, 'SChool of Basic Medical Science, North China University of Science and Technology, Tangshan, eHebei Key Laboratory for Chronic Diseases, Tangshan Key Laboratory for Preclinical and Basic Research on Chronic Diseases, Tangshan, China
\end{abstract}

\section{Key Words}

Traumatic brain injury $\bullet$ Vitamin D $\bullet$ Calcitriol $•$ Autophagy $\bullet$ Apoptosis $•$ Function recovery

\begin{abstract}
Background/Aims: Traumatic brain injury (TBI) is a major public health problem in the world and causes high rates of mortality and disability. Recent evidence suggests that vitamin D (VD) has neuroprotective actions and can promote function recovery after TBI. In vitro and in vivo studies have demonstrated that autophagy could be enhanced following supplementation with an active metabolite of VD (calcitriol). However, it is unclear whether autophagy participates in the protective effects of calcitriol after TBI. To test this hypothesis, we examined the protective effects of calcitriol on TBI-induced neurological impairment and further investigated whether calcitriol could modulate autophagy dysfunction-mediated cell death in the cortex region of rat brain. Methods: Eighty-five male rats $(250-280 \mathrm{~g})$ were randomly assigned to sham $(n=15)$, TBI model (TBI, $\mathrm{n}=35$ ) and calcitriol treatment (calcitriol, $\mathrm{n}=35$ ) groups. Rats were injected intraperitoneally with calcitriol $(1 \mu \mathrm{g} / \mathrm{kg})$ at $30 \mathrm{~min}, 24 \mathrm{~h}$ and $48 \mathrm{~h}$ post-TBI in the calcitriol group. The lysosomal inhibitor, chloroquine (CQ), was used to evaluate autophagic flux in the TBI and calcitriol groups. Neurological functions were evaluated via the modified neurological severity score test at 1-7 days after TBI or sham operation, and the terminal deoxynucleotidyl transferase-mediated FITC-dUTP nick-end labeling method was used to evaluate the ability of calcitriol to inhibit apoptosis. The expression of VDR, LC3 and p62 proteins was measured by western blot analysis at 1, 3 and 7 days post-injury. Results: Calcitriol treatment attenuated mNSS at $2-7$ days post-TBI $(P<0.05$ versus TBI group). Calcitriol dramatically increased VDR protein expression compared with the untreated counterparts at 1,3 and 7 days post-TBI
\end{abstract}

C. Cui and J. Cui contributed equally to the studies and should be considered as co-first authors. 
$(P<0.05)$. The rate of apoptotic cells in calcitriol-treated rats was significantly reduced compared to that observed in the TBI group $(P<0.05)$. The LC3II/LC3I ratio was decreased in the cortex region at 1,3 and 7 days post-TBI in rats treated with calcitriol ( $p<0.05$ versus TBI group), and the p62 expression was also attenuated ( $p<0.05$ versus TBI group). The LC3II/LC3I ratio in the calcitriol group was significantly increased when pretreated with CQ $(P<0.05)$. Conclusion: Calcitriol treatment activated VDR protein expression and attenuated neurological deficits in this rat TBI model. The protective effects might be associated with the restoration of autophagy flux and the decrease in apoptosis in the cortex region of rat brain.

(C) 2017 The Author(s)

Published by S. Karger AG, Basel

\section{Introduction}

Traumatic brain injury (TBI) is a leading cause of mortality in young populations. TBI patients suffer permanent neurological and psychological disabilities that represent a significant social and economic burden [1]. Although considerable progress has been made in animal models and preclinical research in recent years [2-5], there are currently no available therapeutic strategies in clinical practice for TBI. Therefore, finding new, effective therapeutic drugs and developing novel therapeutic strategies are important issues.

There is emerging evidence that nutritional interventions are promising treatment adjuncts, given their documented benefits, favorable safety profiles, ease of accessibility and low cost [6]. Vitamin D (VD), which is most commonly associated with the regulation of calcium homeostasis, exerts its endocrinological influence via the nuclear vitamin $\mathrm{D}$ receptor (VDR) [7-9]. Recently, VD supplementation and VD deficiency have been associated with neuronal injury and function recovery after TBI $[10,11]$. However, the neuroprotective mechanisms of VD are complex and multidimensional [12] and have not been fully addressed.

Autophagy is a cellular fundamental biological process for the degradation of cytoplasmic components such as organelles and proteins aggregates. Several studies have focused on the effect of autophagy in aging, cancer, neurodegeneration disease and acute brain injury [13-16]. Therefore, strategies that regulate autophagy may guard against or cure human disorders [13-16]. Recently, in vitro and in vivo studies have demonstrated that autophagy could be enhanced following supplementation with an active metabolite of VD (calcitriol) $[17,18]$. However, it is unclear whether autophagy participates in the protective effects of calcitriol after TBI.

In the present experiment, through continual treatment with calcitriol in a rat model of TBI, we sought to test the protective effects of the active metabolite of VD against TBIinduced neurological deficits. We further examined whether calcitriol could modulate autophagy dysfunction-mediated cell death in the cortex region of rat brain.

\section{Materials and Methods}

\section{Animals and experimental groups}

All experiments were in compliance with the guidelines of the National Institutes of Health for the Care and Use of Laboratory Animals and approved by the North China University of Science and Technology Experimental Ethics Committee. Because the effects of estrogen on neurological function and menstruation in female rats are irregular, we selected male rats in the present study. Eighty-five male rats (body weight 250-280 g) were supplied by the Experimental Animal Center of North China University of Science and Technology (Tangshan, Hebei, China) and housed in the animal facility under a 12 hour day and night light cycle. They were allowed free access to food and water. All of them were randomly assigned to sham $(n=15)$, TBI model (TBI, $n=35$ ) and calcitriol treatment (calcitriol, $n=35$ ) groups. In the calcitriol group, the rats were injected intraperitoneally with $1 \mu \mathrm{g} / \mathrm{kg}$ calcitriol (Sigma, USA, dissolved in a 5\% DMSO solution) at 30 min, $24 \mathrm{~h}$ and $48 \mathrm{~h}$ post-TBI. At the same times, both sham and TBI groups received equal volumes of DMSO by intraperitoneal injection. To evaluate autophagic flux in vivo, the lysosomal inhibitor, chloroquine (CQ),

\section{KARGER}




\section{Cellular Physiology Cell Physiol Biochem 2017;42:1888-1896 \begin{tabular}{ll|l} 
DOI: 10.1159/000479571 03,2017 & $\begin{array}{l}\text { O 2017 The Author(s). Published by S. Karger AG, Basel } \\
\text { www.karger.com/cpb }\end{array}$ \\
\hline and Biochemistry Published online: August 03,
\end{tabular}}

Cui et al.: Vitamin D Receptor Attenuates Autophagy Dysfunction

was injected (Sigma, USA, dissolved in a 5\% DMSO solution) intraperitoneally at $10 \mathrm{mg} / \mathrm{kg} 1$ hour before TBI induction. At the same time, an equal volume of DMSO was injected for the controls.

TBI model

The rat TBI model was created as described in our previous study [4]. In brief, after inducing anesthesia with an intraperitoneal injection of $10 \%$ chloral hydrate $(3 \mathrm{ml} / \mathrm{kg})$, the head of the animal was fixed on a stereotactic frame. Aseptic techniques were used throughout the surgery. A midline scalp incision was performed to expose the skull. A 6-mm craniotomy was performed over the right parietal cortex, centered on the coronal suture and $2.5 \mathrm{~mm}$ lateral to the sagittal suture (velocity $=5 \mathrm{~m} / \mathrm{s}$, depth $=2.5 \mathrm{~mm}$, and dwell time $=100 \mathrm{~ms}$ ). The bone flap was immediately replaced and sealed, and the scalp was sutured closed. The rectal temperature was maintained at $37^{\circ} \mathrm{C}$ with heating pads and lamps. The animals were returned to the feeding room after recovery from anesthesia. Sham-operated rats underwent procedures identical to those of the TBI animals, including anesthesia and surgery, but without TBI.

\section{Evaluation of neurological function}

Neurological functional measurements were performed using the modified neurological severity score (mNSS) test, which is a composite of the motor, sensory and balance tests and reflex tests. The test was carried out on days 1-14 after TBI or sham operation. One point was scored for the inability to perform each test or for the lack of a tested reflex. Neurological function was graded on a scale of 0-18 (normal score, 0 ; maximal deficit score, 18).

\section{Western blot analysis}

The cortex region of the brain tissue was lysed in tissue protein lysis solution (Life Technologies, Gent, Belgium) containing 5\% proteinase inhibitor cocktail (Sigma Aldrich, Diegem, Belgium), kept on ice for a 30 min incubation, and then centrifuged at 15,000 g for $15 \mathrm{~min}$. The bicinchoninic acid (BCA) protein assay (Jiancheng, Nanjing, China) was used to determine the protein concentration. Proteins from each sample were run on a 10\% SDS-PAGE and transferred to PVDF membranes that were blocked with 5\% BSA for $1 \mathrm{~h}$, followed by overnight incubation at $4{ }^{\circ} \mathrm{C}$ with various antibodies: rabbit anti-rat VDR, LC3, p62 and $\beta$-actin polyclonal antibodies (1:1000 dilution, Santa Cruz, CA, USA). The membranes were washed the next day and incubated in secondary antibodies (1:5000 dilution, Danvers, MA, USA). The bands were visualized with an enhanced chemiluminescent reagent (ECL, Hercules, CA, USA) and quantified using Image Quant 5.2 software (Molecular Dynamics, Sunnyvale, CA, USA).

\section{Assessment of Apoptosis}

The presence of apoptosis in the cortex region of rat brain was assessed by the terminal deoxynucleotidyl transferase-mediated FITC-dUTP nick-end labeling method (TUNEL) following the manufacturer's protocol. Nuclei were counterstained with DAPI. The number of TUNEL-DAPI positive cells was counted as described previously [19]. The counting area was located in the same position in all groups. For each group, quantification was performed in sections from three different rats. Rate of apoptotic cells $=$ TUNEL positive cells / DAPI positive cells $\times 100 \%$.

\section{Statistical analysis}

All data are expressed as the means \pm SD and analyzed using one-way ANOVA followed by the StudentNewman-Keuls post hoc test (SPSS 17.0 software). A statistically significant difference was designated when $P<0.05$.

\section{Results}

Neurological deficits were improved by calcitriol treatment after TBI

To determine the effects of calcitriol treatment on neurological functions, the mNSS was examined 1-7 days after TBI or sham operation. As demonstrated in Fig. 1, TBI induced significant neurological deficits compared with sham-operated animals $(P<0.05)$. Calcitrioltreated rats, on the other hand, displayed improved neurological functions, as evidenced 
by decreased mNSS compared with their untreated counterparts at 2-7 days post-TBI $(P<0.05)$.

Treatment with calcitriol increased VDR expression

Western blotting was performed to detect the expression of VDR protein at 1, 3 and 7 days in TBI or sham-operated rats. As depicted in Fig. 2, compared to the sham group, TBI caused a decrease in VDR expression at 1 and 3 days $(P<0.05)$. Otherwise, treatment with calcitriol dramatically increased VDR protein expression compared with the untreated counterparts at 1,3 and 7 days post-TBI $(P<0.05)$. Time-course studies of VDR expression indicated that VDR was expressed in rat brain tissue and that calcitriol treatment induced VDR expression in the cortex region of TBI rats.

Treatment with calcitriol suppressed apoptosis in the cortex region

To evaluate the ability of calcitriol to inhibit apoptosis, the TUNEL method was used at 3 days after TBI or sham operation. Our results indicated that the rate of apoptotic cells in the cortex was markedly increased after TBI $(P<0.05$ versus sham group), whereas the rate of apoptotic cells in calcitriol-treated rats was significantly reduced compared to that observed in the TBI group $(P<0.05)$ (Fig. 3).

Change in autophagy-related protein expression after calcitriol treatment

Western blotting was performed to detect the LC3II/LC3I ratio and p62 protein expression in the cortex. Compared to the sham group, the LC3II/LC3I ratio was increased in the TBI group at 1-7 days $(P<0.05)$. Calcitriol dramatically decreased the LC3II/LC3I ratio at 1-7 days $(P<0.05$ versus TBI group). Furthermore, it has been proven that the 662 protein interacts with LC3 and ubiquitinated proteins, which can be degraded through autophagy flux [20]. At 1-7 days after TBI, an increased protein level of p62 was detected in the cortex $(P<0.05$ versus sham group). We observed that calcitriol treatment significantly attenuated p62 protein levels compared to the TBI group at 1-7 days $(P<0.05)$ (Fig. 4).

Treatment with CQ abrogated the calcitriol-induced decrease in the LC3II/LC3I ratio

To determine the role of calcitriol treatment in autophagy flux in response to TBI, the LC3-II/LC3-I ratio was evaluated with and without the lysosome inhibitor CQ at 3 days. As

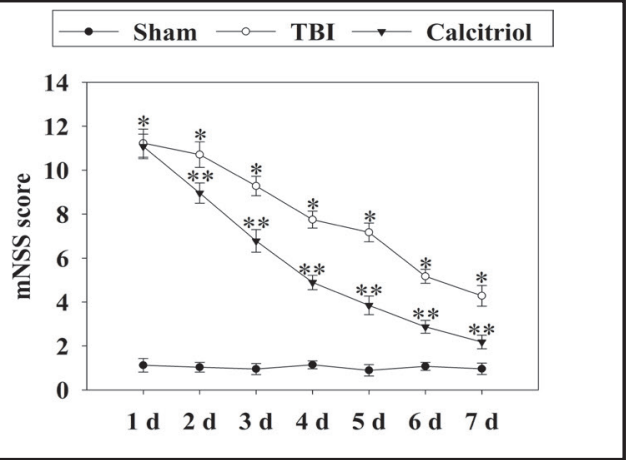

Fig. 1. Neurological deficits were improved by calcitriol treatment after TBI. mNSS was examined to detect the neurological functions at 1-7 days after TBI or sham operation in rats ( $n=5$, per group). TBI induced significant neurological deficits compared with sham-operated animals at all time points analyzed after injury $\left({ }^{*} P<0.05\right)$. The mNSS was significantly lower in the calcitriol group than the TBI group at $2-7$ days $\left({ }^{* *} P<0.05\right)$.

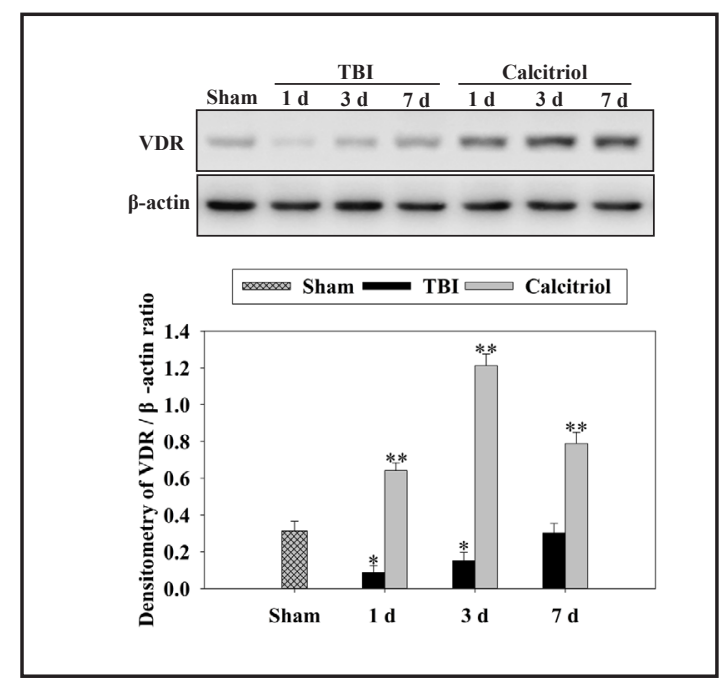

Fig. 2. Increase in VDR expression after calcitriol treatment. Western blotting was performed to detect the VDR expression at 1, 3 and 7 days in TBI or sham-operated rats. Densitometry analysis of VDR bands corresponding to $\beta$-actin. Bars represent the mean \pm standard error ( $n=5$, per time point). ${ }^{*} p<$ 0.05 versus sham group, ${ }^{* *} \mathrm{p}<0.05$ versus TBI group. 
Fig. 3. Treatment with calcitriol suppressed apoptosis in the cortex region. Apoptotic cell death was assessed by DAPI and TUNEL staining at 3 days (scale bar, $50 \mu \mathrm{m}$ ). Representative confocal images stained for TUNEL (green) and DAPI (blue) showed the effect of calcitriol on TBI-induced cell apoptosis. Bars represent statistical analysis of the relative apoptotic cell rate. Data are presented as the mean \pm standard error ( $\mathrm{n}=5$, per group). ${ }^{*} \mathrm{p}<0.05$ versus sham group, ${ }^{* *} \mathrm{p}<0.05$ versus TBI group.

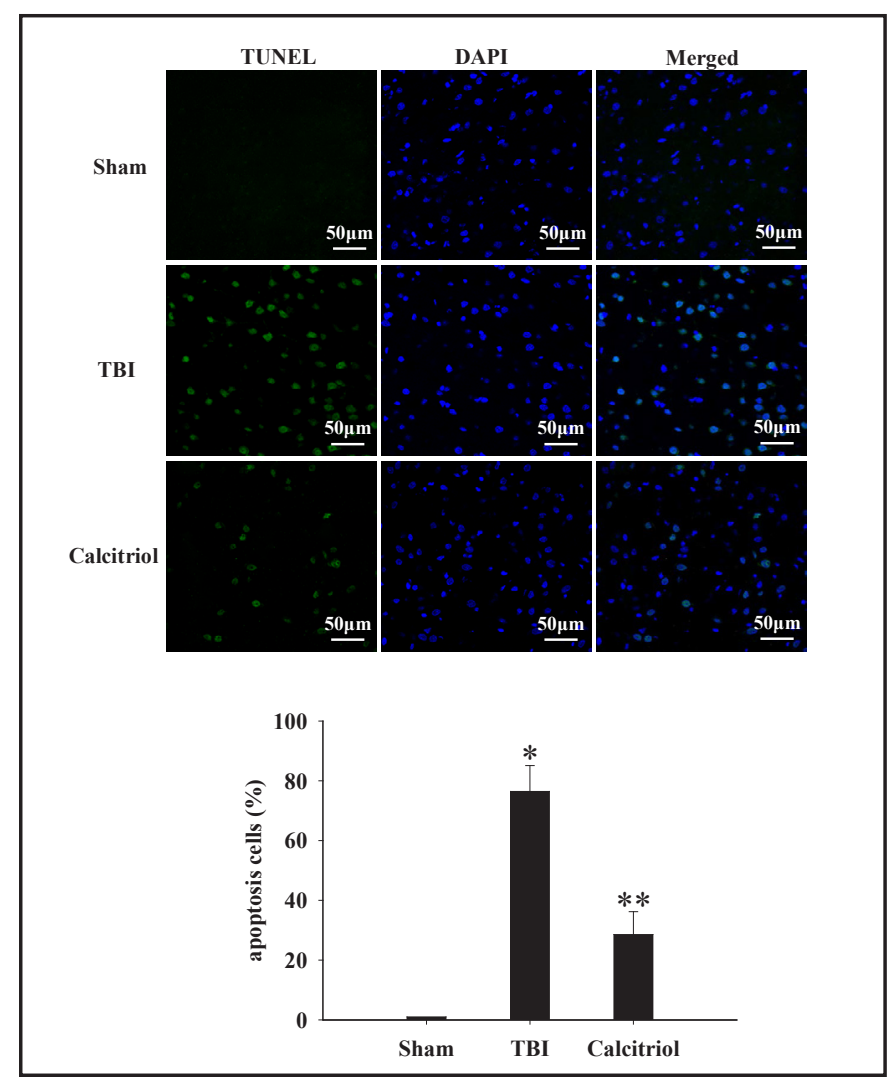

Fig. 4. Change in autophagy-related protein expression after calcitriol treatment. Western blot analysis demonstrates the LC3II/LC3I ratio and p62 levels at 1, 3 and 7 days in TBI or sham-operated rats. The quantitative results of p62 are expressed as the ratio of the densitometries to $\beta$-actin bands. Values are expressed as the mean \pm standard error $(n=5$, per time point). ${ }^{*} p<0.05$ versus sham group, ${ }^{* *} \mathrm{p}<0.05$ versus TBI group.

depicted in Fig. 5, CQ administration did not induce an additional increase in the LC3II/ LC3I ratio in rats in the TBI group $(P>0.05)$. Nevertheless, the LC3II/LC3I ratio in the calcitriol group was significantly increased when pretreated with CQ $(P<0.05)$ (Fig. 5).

\section{Discussion}

It is well known that TBI can result in significant neurological deficits in humans [1]. The purpose of the current study was to assess the neuroprotective effects of calcitriol after TBI. The present study demonstrated

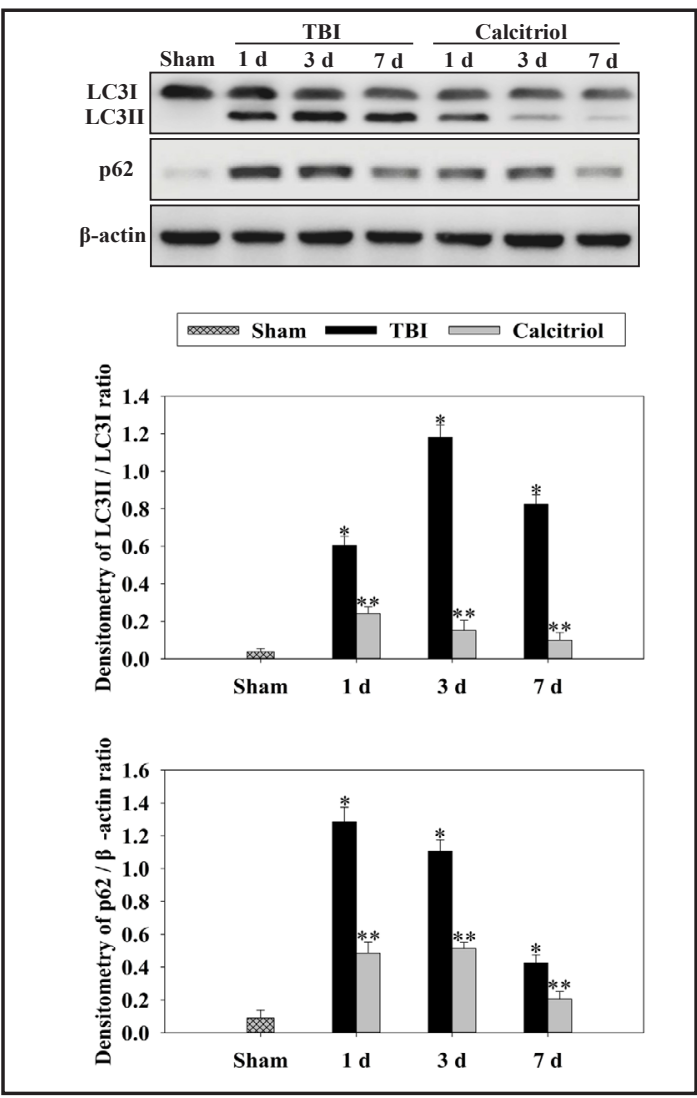


Fig. 5. Preventative treatment with $C Q$ induced an additional increase in the LC3II/LC3I ratio in calcitriol-treated rats. LC3-II/LC3-I ratios were evaluated with and without CQ at 3 days in the TBI and calcitriol groups. Values are expressed as the mean \pm standard error ( $n=5$, per group). ${ }^{*} \mathrm{p}<0.05$.

that post-TBI administration of calcitriol could improve the recovery of behavioral deficits. At the molecular level, the TUNEL method was used to evaluate the protective ability of calcitriol against cell death at 3 days. We found that the rate of apoptotic cells in the cortex region was markedly reduced in the calcitriol group compared with the TBI group, indicating that calcitriol inhibits cell death induced by TBI. Furthermore, previous studies have demonstrated that calcitriol could exert neuroprotective actions in

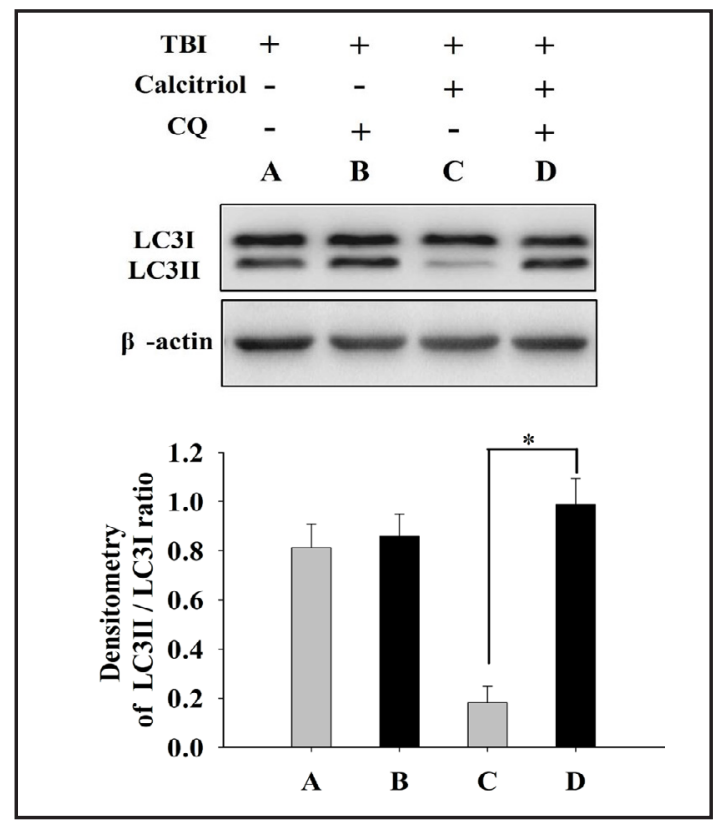
multiple models of acquired brain injury, such as traumatic brain injury, stroke, spinal cord injury and Parkinson's disease [18, 21, 22]. These findings, together with the observations of the present study, may aid in improving the current understanding of calcitriol-mediated neuroprotection in neurological disorders.

Nuclear hormone receptors are a family of transcription factors involved in diverse physiological functions [23]. The VDR is a member of this superfamily and is widely distributed in the central nervous system, regulating multiple aspects of neuronal functions $[7,8]$. In the present study, VDR expression in the cortex region was significantly elevated following calcitriol treatment. The pathological processes caused by TBI include primary and secondary injury mechanisms [24]. Primary damage is due to immediate brain tissue disruption. In comparison, secondary injury is delayed and is produced via complicated processes, such as $\mathrm{Ca}^{2+}$ influx, oxidative stress and inflammatory response [24]. VDR activation post-TBI suppressed intracellular $\mathrm{Ca}^{2+}$ through increased intracellular $\mathrm{Ca}^{2+}$ buffering and decreased L-type voltage-sensitive $\mathrm{Ca}^{2+}$ channels, which caused a reduction in discriminate glutamate release and resultant neurotoxicity [25]. Cekic et al have found that the activation of the VDR attenuated the inflammatory response induced by TBI [26]. Moreover, Kalueff et al have shown that VDR activation up-regulated free radical scavenging and down-regulated oxidative stress [27]. This pathogenesis ultimately leads to neuronal apoptosis. In agreement with previous studies, we also observed that activation of the VDR suppressed high rates of TBI-induced cell apoptosis, further supporting the notion that the VDR acts as a self-defensive protein to overcome the acute pathological stress in TBI.

Our study also adds novel evidence that calcitriol treatment significantly maintains autophagy flux for 1-7 days post-TBI. Autophagy maintains cellular homeostasis by the selective degradation of cytoplasmic components [13-16]. It is related to the pathology of many neurological diseases in the central nervous system, such as Alzheimer's disease, ischemic brain damage, subarachnoid hemorrhage, spinal cord injury and TBI [4, 28-31]. A mammalian homolog of Atg8, LC3, is found exclusively in the autophagosomal membrane and is widely used as an autophagic marker to monitor the formation and activity of autophagy [32]. To further confirm that treatment with calcitriol was involved in the regulation of autophagic activity, we measured the conversion of the soluble form of LC3 (LC3I) to the lipidated and autophagosome-associated form (LC3II). We found that the ratio of LC3II/ LC3I was significantly increased at 1-7 days post-TBI, indicating stimulated autophagy and increased autophagosome abundance. However, preventing autophagosome-lysosome fusion with CQ did not induce an additional increase in LC3II/LC3I, indicating that the 
increased ratio of LC3II/LC3I in the TBI group resulted from the dysfunction of autophagy and the impairment of autophagosome clearance. Moreover, calcitriol treatment induced a decrease in the LC3II/LC3I ratio accompanied by a decrease in p62, a specific autophagic substrate protein and hallmark of autophagic flux [20]. We also observed that pretreatment with CQ significantly increased the LC3II/LC3I ratio in calcitriol-treated rats. These results suggest that the protection effect of calcitriol is related to the restoration of autophagy flux and thereby inhibits autophagy dysfunction. Our findings are similar to those of Yao et al [33], who demonstrated that in vivo activation of the VDR protected against myocardial reperfusion injury through restoring autophagy flux and inhibiting apoptosis. Therefore, it is conceivable that the restored autophagy flux induced by VDR activation may play an essential role in the homeostatic balance between cellular death and survival after TBI.

However, a limitation of the present study is the exclusion of female rats. It should be noted that both estrogen and progesterone could affect TBI-induced damage [34]. Previous studies have demonstrated the interactions between estrogen and VD in human umbilical artery vascular smooth muscle cells and spermatozoa functions [35, 36]. Moreover, a combination of calcitriol and progesterone can improve pathological outcomes after TBI, with a varying response to the calcitriol dose [11]. Thus, it would be interesting to verify the protection mechanism of calcitriol in females in a future study.

\section{Conclusion}

In summary, the present study demonstrated that calcitriol treatment activated the VDR pathway and attenuated neurological deficits in a rat TBI model. The neuroprotective effects might be derived from the calcitriol-induced restoration of autophagy flux and antiapoptotic effects in the cortex region of rat brain.

\section{Disclosure Statement}

None.

\section{References}

1 Asemota AO, George BP, Bowman SM, Haider AH, Schneider EB: Causes and trends in traumatic brain injury for United States adolescents. J Neurotrauma 2013;30:67-75.

-2 Mustafa G, Hou J, Tsuda S, Nelson R, Sinharoy A, Wilkie Z, Pandey R, Caudle RM, Neubert JK, Thompson FJ: Trigeminal neuroplasticity underlies allodynia in a preclinical model of mild closed head traumatic brain injury (cTBI). Neuropharmacology 2016;107:27-39.

-3 Cui C, Cui Y, Gao J, Sun L, Wang Y, Wang K, Li R, Tian Y, Song S, Cui J: Neuroprotective effect of ceftriaxone in a rat model of traumatic brain injury. Neurol Sci 2014;35:695-700.

4 Feng Y, Gao J, Cui Y, Li M, Li R, Cui C, Cui J: Neuroprotective effects of resatorvid against traumatic brain injury in rat: involvement of neuronal autophagy and TLR4 signaling pathway. Cell Mol Neurobiol 2016:1-14.

5 Feng Y, Cui Y, Gao JL, Li MH, Li R, Jiang XH, Tian YX, Wang KJ, Cui CM, Cui JZ: Resveratrol attenuates neuronal autophagy and inflammatory injury by inhibiting the TLR4/NF- $\kappa B$ signaling pathway in experimental traumatic brain injury. Int J Mol Med 2016;37:921-930.

6 Scrimgeour AG, Condlin ML: Nutritional treatment for traumatic brain injury. J Neurotrauma 2014;31:989999.

7 Jiang P, Xue Y, Li HD, Liu YP, Cai HL, Tang MM, Zhang LH: Dysregulation of vitamin D metabolism in the brain and myocardium of rats following prolonged exposure to dexamethasone. Psychopharmacology $2014 ; 231: 3445-3451$. 


\section{Cellular Physiology Cell Physiol Biochem 2017;42:1888-1896 \begin{tabular}{l|l} 
DOI: 10.1159/000479571 03,2017 & $\begin{array}{l}\text { O 2017 The Author(s). Published by S. Karger AG, Basel } \\
\text { www.karger.com/cpb }\end{array}$ \\
\hline and Biochemistry Published online: August 03,
\end{tabular}}

8 Jiang P, Zhang WY, Li HD, Cai HL, Liu YP, Chen LY: Stress and vitamin D: Altered vitamin D metabolism in both the hippocampus and myocardium of chronic unpredictable mild stress exposed rats. Psychoneuroendocrinology 2013;38:2091-2098.

-9 He XJ, Yan D, Wei X, Dang XQ: Roles of 1,25(OH)2D3 and vitamin D receptor in the pathogenesis of rheumatoid arthritis and systemic lupus erythematosus by regulating the activation of CD4+ T cells and the PKC $\delta /$ ERK signaling pathway. Cell Physiol Biochem 2016;40:743-756.

10 Cekic M, Cutler SM, Vanlandingham JW, Stein DG: Vitamin D deficiency reduces the benefits of progesterone treatment after brain injury in aged rats. Neurobiol Aging 2009;32:864-874.

11 Hua F, Reiss JI, Tang H, Wang J, Fowler X, Sayeed I, Stein DG: Progesterone and low-dose vitamin D hormone treatment enhances sparing of memory following traumatic brain injury. Horm Behav 2012;61:642-651.

12 Lawrence DW, Sharma B: A review of the neuroprotective role of vitamin D in traumatic brain injury with implications for supplementation post-concussion. Brain Inj 2016;30:960-968.

13 Rubinsztein DC, Mariño G, Kroemer G: Autophagy and aging. Cell 2011;146:682-695.

14 Jin S, White E: Tumor suppression by autophagy through the management of metabolic stress. Autophagy 2008;4:563-566.

15 Nixon RA: The role of autophagy in neurodegenerative disease. Nat Med 2013;19:983-997.

16 Galluzzi L, Bravo-San Pedro JM, Blomgren K, Kroemer G: Autophagy in acute brain injury. Nat Rev Neurosci 2016;17:467-484.

17 Wang RC, Levine B: Calcipotriol induces autophagy in HeLa cells and keratinocytes. J Invest Dermatol 2011;131:990-993.

18 Zhou KL, Chen DH, Jin HM, Wu K, Wang XY, Xu HZ, Zhang XL: Effects of calcitriol on experimental spinal cord injury in rats. Spinal Cord 2016;54:510-516.

19 Wang P, Wu Q, Wu W, Li H, Guo Y, Peng Y, Gao G, Shi Z, Zhao B, Chang YZ: Mitochondrial ferritin deletion exacerbates $\beta$-amyloid-induced neurotoxicity in mice. Oxid Med Cell Longev 2017;2017:1020357.

-20 Pankiv S, Clausen TH, Lamark T, Brech A, Bruun JA, Outzen H, A Ø, Bjørkøy G, Johansen T: p62/SQSTM1 binds directly to Atg8/LC3 to facilitate degradation of ubiquitinated protein aggregates by autophagy. J Biol Chem 2007;282:24131-24145.

21 Tang H, Fang H, Wang J, Yousuf S, Atif F, Sayeed I, Stein DG: Progesterone and vitamin D combination therapy modulates inflammatory response after traumatic brain injury. Brain Inj 2015;29:1-10.

-22 Fu J, Xue R, Gu J, Xiao Y, Zhong H, Pan X, Ran R: Neuroprotective effect of calcitriol on ischemic/reperfusion injury through the NR3A/CREB pathways in the rat hippocampus. Mol Med Rep 2013;8:1708-1714.

23 Aranda A, Pascual A: Nuclear hormone receptors and gene expression. Physiol Rev 2001;81:1269-1304.

24 Ray SK, Dixon CE, Banik NL: Molecular mechanisms in the pathogenesis of traumatic brain injury. Histol Histopathol 2002;17:1137-1152.

25 Brewer LD, Thibault V, Chen KC, Langub MC, Landfield PW, Porter NM: Vitamin D hormone confers neuroprotection in parallel with downregulation of L-type calcium channel expression in hippocampal neurons. J Neurosci 2001;21:98-108.

-26 Cekic M, Sayeed I, Stein DG: Combination treatment with progesterone and vitamin D hormone may be more effective than monotherapy for nervous system injury and disease. Front Neuroendocrinol 2009;30:158172.

-27 Kalueff AV, Eremin KO, Tuohimaa P: Mechanisms of neuroprotective action of vitamin D(3). Biochemistry 2004;69:738-741.

28 Tung YT, Wang BJ, Hu MK, Hsu WM, Lee H, Huang WP, Liao YF: Autophagy: a double-edged sword in Alzheimer's disease. J Biosci 2012;37:157-165.

29 Sheng R, Zhang LS, Han R, Liu XQ, Gao B, Qin ZH: Autophagy activation is associated with neuroprotection in a rat model of focal cerebral ischemic preconditioning. Autophagy 2010;6:482-494.

-30 Yan F, Li J, Chen J, Hu Q Gu C, Lin W, Chen G: Endoplasmic reticulum stress is associated with neuroprotection against apoptosis via autophagy activation in a rat model of subarachnoid hemorrhage. Neurosci Lett 2014;563:160-165.

-31 Sekiguchi A, Kanno H, Ozawa H, Yamaya S, Itoi E: Rapamycin promotes autophagy and reduces neural tissue damage and locomotor impairment after spinal cord injury in mice. J Neurotrauma 2012;29:946-956.

-32 Kabeya Y, Mizushima N, Ueno T, Yamamoto A, Kirisako T, Noda T, Kominami E, Ohsumi Y, Yoshimori T: LC3, a mammalian homologue of yeast Apg8p, is localized in autophagosome membranes after processing. EMBO J 2000;22:5720-5728. 


\section{Cellular Physiology Cell Physiol Biochem 2017;42:1888-1896 \begin{tabular}{ll|l} 
and Biochemistry 10.1159/000479571 & $\begin{array}{l}\text { O 2017 The Author(s). Published by S. Karger AG, Basel } \\
\text { wwww.karger.com/cpb }\end{array}$ \\
\cline { 2 - 3 }
\end{tabular}}

Cui et al.: Vitamin D Receptor Attenuates Autophagy Dysfunction

33 Yao T, Ying X, Zhao Y, Yuan A, He Q Tong H, Ding S, Liu J, Peng X, Gao E: Vitamin D receptor activation protects against myocardial reperfusion injury through inhibition of apoptosis and modulation of autophagy. Antioxid Redox Signal 2014;22:633-650.

-34 Khaksari M, Soltani Z, Shahrokhi N, Moshtaghi G, Asadikaram G: The role of estrogen and progesterone, administered alone and in combination, in modulating cytokine concentration following traumatic brain injury. Can J Physiol Pharmacol 2011;89:31-40.

-35 Somjen D, Knoll E, Sharon O, Many A, Stern N: Interaction between the effects of the selective estrogen modulator femarelle and a vitamin D analog in human umbilical artery vascular smooth muscle cells. J Steroid Biochem Mol Biol DOI: 10.1016/j.jsbmb.2017.05.007.

-36 Zanatta AP, Brouard V, Gautier C, Goncalves R, Bouraïma-Lelong H, Silva FRMB, Delalande C: Interactions between oestrogen and $1 \alpha, 25(\mathrm{OH}) 2$-vitamin D 3 signalling and their roles in spermatogenesis and spermatozoa functions. Basic Clin Androl DOI:10.1186/s12610-017-0053-z. 